\title{
Masonry Arch Technology Mortar Joint Control Method and Stress
}

\author{
Analysis \\ Yan Weiya ${ }^{1, a}$ \\ ${ }^{1}$ College of Civil and Architectural Engineering of Taiyuan University of Technology, No.79, West \\ Yingze Avenue,Wanbailin Distrct, Taiyuan City,Shanxi Province,P.R. China 030024, China \\ atjywy@qq.com
}

Keywords:Masonry arch;Masonry method;Mortar joint control;Application of arch

\begin{abstract}
The arch is the most commonly used in wood structure building form China in ancient architecture, the technical requirements and unique characteristics of Chinese space is an important part of ancient architecture.In this paper, through some simple calculation formula, simply introduces the masonry arch, summarizes the application scope of arch of the technical characteristics and masonry arch technology in the China.
\end{abstract}

\section{The development of the arch structure}

The building with the arch type structure is mainly built of brick, stone or adobe material。 Cross space structure of brick masonry from bond with these materials, not only covers the lower space, but also played a role in supporting the enclosing walls, and on the roof. Arch technology first began in the 4000 BC in Mesopotamia Area, and had a large-scale application in the ancient buildings in Rome.In order to cross bending force into pressure in the space structure , and Brick arch shaped.Multi arch parallel constitutes a barrel arch form, and will each arch masonry are mutually staggered, constitute one connected in the structure of the longitudinal barrel arch.A layer of flat brick or stone in the brick arch, makes the arch structure stronger .The Song Dynasty call this layer of masonry "Jiao Bei", the Qing Dynasty called "Fu" 。 The heavy structure of the upper load is need to use several layers of arch and "Fu" overlapping sets, in order to enhance the strength of the structure.

The curvature of the arch structure issue early slowly,Rise and arch span ratio is less than 0.5,Then the high span ratio become $0.5 \mathrm{arch}$, forming a standard semicircular arch. Since the Ming Dynasty, with the development and popularization of the technology of masonry, building arch technique also improves.High span ratio greater than 0.5 (three heart arch) has been more common.Provisions in the Qing Dynasty "engineering practice" in the form of building arch high span ratio is 0.55 . The higher proportion of arch form, not only the stress is more reasonable, and the vision is very stretch and suitable, appropriate examples is combined with the structure and shape proportion of Art. 


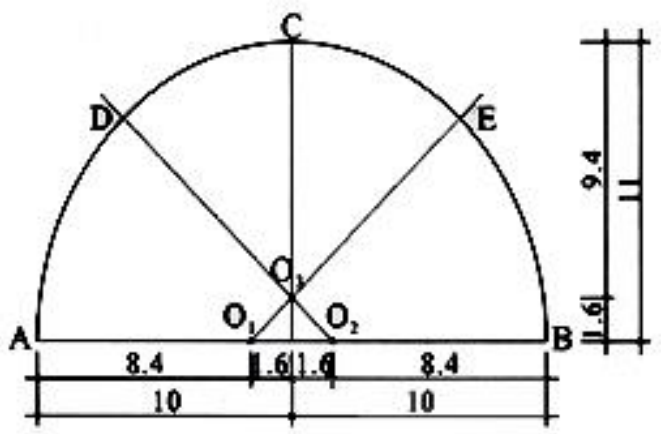

Fig1:Three circle arch practice

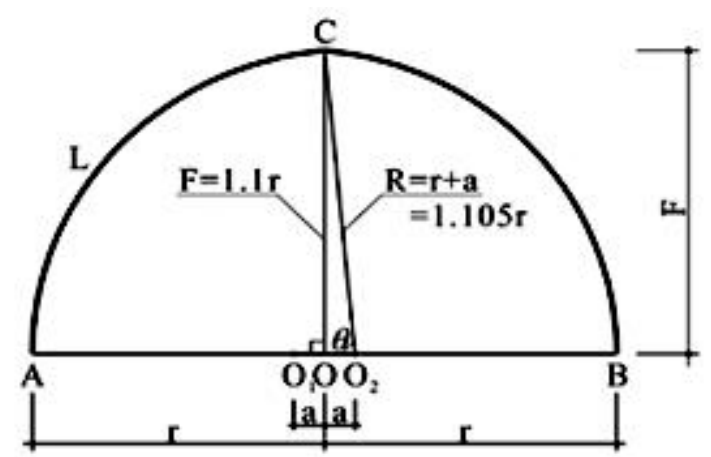

Fig2:Double circle arch practice

\section{Simple Arching method of double heart round masonry arch}

Cave, culvert or courtyard gate building, often using the way of masonry arch .Top of Double heart round arch is slightly higher than the top of the semi-circular arch.The specific practices are as follows:

In order to make the top of arch slightly pointed than the semicircular top, Can take a short distance respectively in the bottom edge of the midpoint(the midpoint circle diameter,hypothesize the center is $\mathrm{O}$ ) on both sides of the semicircle arc, And make the $\mathrm{O}_{1} \mathrm{O}$ equal to $\mathrm{O}_{2} \mathrm{O}$ ( The dashed

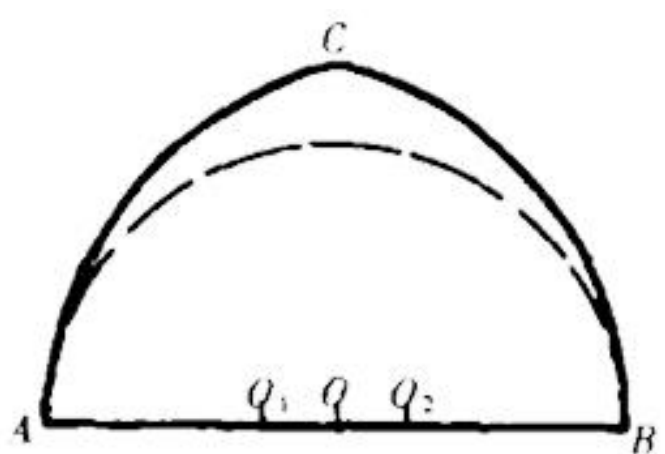

Fig3:Masonry arch camber sketch line is the semi-circular arch In the following illustration), and with $\mathrm{O}_{1} \mathrm{~B}$ as radius , centered on $\mathrm{O}_{1}$ to draw the right half arc BC. then with $\mathrm{O}_{2} \mathrm{~A}$ as radius, centered on $\mathrm{O}_{2}$ to Draw the left half arc AC. According to the arc AC and BC masonry arch.This can get a parabolic arch shape.If you want to make the top of the arch more pointed, can make $\mathrm{O}_{1} \mathrm{O}$ and $\mathrm{O}_{2} \mathrm{Olarger}$; On the other hand, can make the $\mathrm{O}_{1} \mathrm{O}$ and $\mathrm{O}_{2} \mathrm{O}$ shorter.In addition to the double heart round ticket, commonly used stamps shape are round, round three heart and parabola form.

\section{Mortar joint control of the Arch masonry}

Use common brick masonry arch, in addition to guarantee the radian of arch, ensure the strength of the masonry mortar and mortar plumpness, an important one, is to strictly control the uniformity and size in the masonry mortar joint.Different from ordinary brick clay brick masonry arch construction, the requirements of vertical mortar joint, which is an isosceles trapezoid shape basically the same size.Experienced bricklayer,in mortar joint control of the Arch masonry mortar joint control of the flue or tunnel kiln, etc.,often use mention seam practices, there is some truth.However, if improper operation is easy to cause uneven mortar joint. The results directly affect the quality and appearance of the arch masonry. 
To make the arch mortar joint uniform, must first know each mortar joint size. To calculate each mortar joint size must first determine the in seam thickness. And the calculation of the thickness of mortar joint internal and external difference(the difference on both sides of the isosceles trapezoid ).When mortar joint thickness of inside and outside is determined, control the size in construction and timely check on it.

mortar joint calculation procedure is as follows:

Set arch diameter is $\mathrm{D}_{1}$, Single arch diameter is $\mathrm{D}_{2}$, The inner arch perimeter is $C_{1}$, The outer arch perimeter is $C_{2}$, the number of brick in Each arch for N,Arch internal mortar joint thickness is $d_{1}$,As shown in Figure:

First determine the thickness of $d_{1}$, when the $d_{1}$ is determined, you can find the $\mathrm{N}$.

$$
\begin{aligned}
& \mathrm{N}=\pi \mathrm{D}_{1} /\left(\mathrm{d}_{1}+53\right) ; \\
& \mathrm{D}_{2}=\mathrm{D}_{1}+230(\mathrm{~mm}) ; \\
& \mathrm{C}_{1}=\pi \mathrm{D}_{1}(\mathrm{~mm}) ; \\
& \mathrm{C}_{2}=\pi \mathrm{D}_{2}=\pi \mathrm{D}_{1}+\pi \times 230(\mathrm{~mm}) .
\end{aligned}
$$

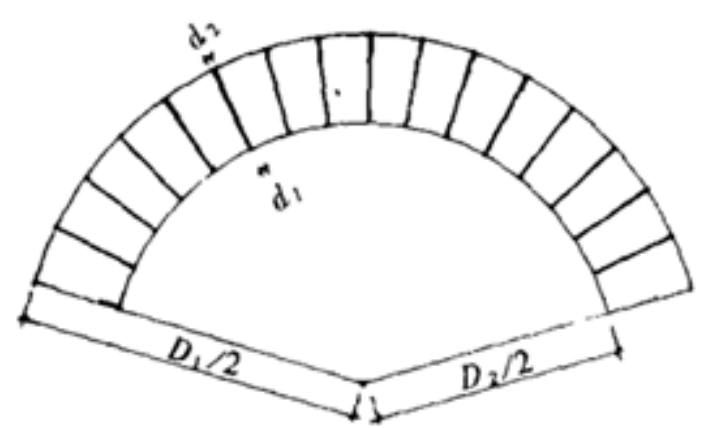

Fig4:Calculation diagram

The difference between the inner and outer arch perimeter $\left(C_{1}-C_{2}\right)$, is the total thickness difference of internal and external mortar joint .

$$
\mathrm{C}_{2}-\mathrm{C}_{1}=\pi \times 230=722(\mathrm{~mm}) ;
$$

Each mortar joint thickness difference for inside and outside:

$$
\left(\mathrm{C}_{2}-\mathrm{C}_{1}\right) / \mathrm{N}=722 / \mathrm{N}(\mathrm{mm})
$$

According to the regulations, Width of Mortar joint in arch bottom,should be 5 8 mm.Therefore, when calculate the mortar joint thickness, the calculated values should be able to meet the above requirements, otherwise it Should be adjusted.

\section{Summary}

In the history of Western architecture, the arch structure in ancient Rome period once obtained the unprecedented development, and become the most significant characteristics and achievements of the architecture of ancient Rome, a tremendous impact on future generations of European architecture.In ancient China,because the timber frame structure system has been the construction of the mainstream. Although the masonry arch technology have been appeared in the Han Dynasty, but not has been widely used in western architecture .

Chinese ancient artisans also summed up a lot of masonry arch masonry method, by using the properties of masonry material, make the structure more reasonable and more suitable for the architectural form. Main building of Chinese ancient buildings although is mostly wooden buildings, although the development of masonry buildings is not so grand than the 
Europe .In Chinese in ancient architecture,Masonry arch is still widely used in many fields, and the development is not backward than the brick masonry structure in Europe.

\section{References}

[1]Wang Qiheng.Double circle:the basic form of the Qing Dynasty $\operatorname{arch}[\mathrm{J}]$.Ancient garden technology,2013(01)

[2]Ha Yang.Brick and stone architecture art[D].Chongqing University,2001

[3]Fang Yong.China building fifteen talk[M].Peking University press.2010

[4]Liu Xujie.History of ancient Chinese building.The first volume[M].Chinese Building Industry Press. 2009

[5]Han Debao.Chinese architectural culture lecture[M].Life $\bullet$ study $\bullet$ knowledge bookstore .2006 